\title{
Requirements for IMS services and applications over interoperable broadband Public Protection \& Disaster Relief Networks and Commercial Communication Networks
}

\author{
Henryk Gierszal \\ Adam Mickiewicz \\ University, Umultowska \\ 85, 61-614 Poznań, \\ Poland \\ Email: \\ gierszal@amu.edu.pl
}

\author{
Anna Stachowicz, \\ Filip Majerowski, \\ Bartłomiej Kowalczyk, \\ Michał Goryński \\ ITTI, Rubież 46, 61-612 \\ Poznań, Poland \\ Email: $\{$ AStachowicz, \\ FMajerowski, \\ BKowalczyk, \\ MGorynski\}@itti.com.pl
}

\author{
Vassilis Kassouras \\ $\mathrm{KeMeA}$, \\ 4 P. Kanellopoulou, \\ Athens 101 77, Greece \\ Email: \\ kassouras@gmail.com
}

\author{
Spase Drakul \\ THYIA Technologies \\ Sarl, Chemin de Roilbot \\ 19, Pregny-Chambésy, \\ Switzerland \\ Email: \\ drakul@bluewin.ch
}

\begin{abstract}
The paper presents requirements related to a heterogeneous interoperable and transportable gateway called HitGW that is designated to connect different incompatible communication systems used by First-Responders (FRs) and to make them interoperable with IP-based networks. The HitGW allows IMS type of services and applications over a broadband network composed of ordinary Public Protection and Disaster Relief (PPDR) networks and Commercial Communication Networks (CCNs). Such a system solution for FRs becomes indispensable for interoperable, effective, secure, and safety communications especially in national and cross-border crisis scenarios. The innovativeness in the chosen approach to design and implement such a system gateway primary consists in the user needs and current trends of PPDR networks toward 4G LTE type of networks and services.
\end{abstract}

\section{INTRODUCTION}

$\mathrm{T}$ HE purpose of this document is to specify and describe some gathered user requirements in scope of the Hit-Gate (Heterogeneous Interoperable Transportable GATEway) project and to present a system concept how to use IP Multimedia Sub-System (IMS) as a component of the gateway for First-Responders. The main objective of the Hit-Gate project is to develop a generic gateway that allows communications across networks currently used by FRs in Europe especially during crisis events. Nowadays PSC (Public Safety Communication) systems use a large number of different and incompatible technologies therefore compromising or even disabling efficient coordination of combined operations (such as cross-border or crisis management when many services/utilities are involved) [1], [8], [13]

System solutions targeted by the Hit-Gate project are intended to be used by FRs and public safety entities, that is why they should be desirable and approved by the end-users and domain experts. To specify Hit-Gate user requirements, research in the area of general requirements for PSC systems have been performed first. Next, basing on identified and

This work is done within EU $7^{\text {th }}$ Frame Programme - FP7 SECURITY, no. 284940 gathered requirements, they have been presented and consulted with end-users and domain experts in the form of questionnaire, brain storm discussion, and available references (state-of-the-art).

To prepare a list of end-user requirements we have applied VOLERE ([2], [3]) methodology that was however empowered by interactions and feedback from end-users. It allows the requirements to be more objective. The requirements have been defined based on consortium knowledge and experience, end-users/FRs and domain experts' opinions, available knowledge about general requirements for PSC systems, as well as literature survey. A collecting process included desktop research in the area of Public Protection and Disaster Relief (PPDR) systems, questionnaire for end-users and domain experts, and internal consortium discussions. It allowed grouping and prioritizing identified requirements.

For development of an interoperable gateway that meets end-users' and other system requirements the proposed solution is based on the 3GPP IMS architecture where Session Initiation Protocol (SIP) is used for control and signaling and Real-time Transport Protocol (RTP) for delivering voice, messages, and video over IP-based networks.

The paper is organized in the following manner. In Section II a short description of the Hit-Gate project and main features of HitGW are presented. The methodology of requirement collection and identification is described in Section III. Section IV includes general objectives of the Hit-Gate project which have great influence on requirements specification, while Section V comprises main requirements for the Hit-Gate system. The system architecture is demonstrated in Section VI. A trial implementation of the IMS platform for GSM network based on the proposed system architecture is presented in Section VII. In Section VIII conclusions are given. 


\section{II.HIT-GATE PROJECT}

The interoperability problem was perceived by EC a few years ago [19]. The main emphasis was put on spectrum interoperability across Europe ([20], [21], [24]) for broadband PPDR networks [22] that meet users' requirements [23]. These efforts have been also taken by many EU FP7 projects which aim at developing solutions that can deliver the interoperability among different PPDR systems. An objective of some projects is also to define a roadmap towards interoperability for PPDR agencies in Europe to support any cross-border events.

The Hit-Gate project is an EU FP7 SECURITY two-year small or medium-scale focused research project. Project Consortium is composed of eleven organizations from eight European countries ([18]). It includes large companies, Research and Technology Organizations (RTO), Small and Medium Enterprises (SME), and an external community of end-users. Consortium experience and knowledge in the area of development of PSC and security (mission critical) systems together with end-users support enables to develop solution, which is adequate to today's needs and covers lacks of interoperable technologies that offer interoperable IMS services. The main project goal is to develop a novel system solution for interoperability between access network elements (NE) (mobile terminals, base stations) of the FirstResponder Networks (FRNs) and access network elements of the existing CCNs without modifications of handset devices or communication infrastructure of both network types. Because at both European and national/domestic levels, public-safety organizations have adopted a variety of systems, equipment, and incompatible technologies (different technologies, standards, and proprietary solutions of the system manufactures). To answer the FRs needs, HitGW is developed to support all those incompatible technologies ranging from legacy-PMR (Professional Mobile Radio), TETRA (TErrestrial Trunked RAdio) and TETRAPOL, to next-generation networks (e.g. LTE and LTE-Advanced). Other types of networks like GSM (2G), UMTS (3G), 4G LTE, WiFi and WiMAX can be used together with FRNs. Moreover, current security and emergency activities frequently involve multi-national FR teams (e.g., natural crisis response and cross-border operations) [10], [11], [12], thus the HitGW will enable efficient coordination of operations involving more than one nation. The main goal of this system gateway is to allow interoperability among NEs of the FRNs and CCNs used for PSC. It should:

- ensure mission critical requirements of PSC applications, i.e. high-availability, dependability, and security;

- be rapidly deployable over mobile, highly-dynamic, and unpredictable environments where existing infrastructures may be degraded and/or destroyed [4].

The Hit-Gate (HG) framework also includes system requirements and specifications, system architectures, development of the HitGW subsystems, system integration and validation, demonstration of the Hit-Gate Network (HGN), and standardization.

\section{MEthodology For GATHERING ReQuirementS}

In order to gather the requirements from the consortium and end-users involved in Hit-Gate project, it has been considered necessary to use a suitable methodology. Thus, the VOLERE methodology has been chosen as a starting point for this work, adapting its requirements specification template to the particularities and needs of Hit-Gate.

Each of the partners involved in the project follows their own processes in the requirements definition phase performed within their activities. However it was important to propose a common way to formalize the requirements that is easy to use and adapt to the needs of the project by all partners. In this context, VOLERE is a straightforward methodology that does not require a complex analysis to be applied. Furthermore, it guarantees the participation of all relevant actors, who are further involved in the design and development that have to meet the requirements defined.

The adapted methodology allows for identification and formalization of unambiguous requirements, as well as assessment the correctness of the requirement in order to avoid of a lack of completeness and coherence.

The use of a common methodology to gather, classify, and assess the requirements a priori was important. The management of the requirements depends on this common methodology, providing the means to trace the identification, definition, assessment, formalization, and if necessary improvement of the requirements gathered.

On the other hand, the requirements should be the key to evaluate the entire project at the end of the development phase. A set of well-defined and unambiguous requirements is needed, not only as input for any further specifications and development, but also as part of the evaluation framework.

VOLERE defines the gathering process and the shell to register the requirements, classified in 27 categories in 5 main groups:

1. Project drivers, the business-related forces. For example, the purpose of the project is a project driver, as are all of the stakeholders - each for different reasons.

2. Project constraints, restrictions on how the product must be designed. For example, it might have to be implemented in the hand-held device being given to major customers, or it might have to use the existing servers and desktop computers, or any other hardware, software, or business practice.

3. Functional requirements, the fundamental or essential subject matter of the product. They describe what the product has to do or what processing actions it is to take.

4. Nonfunctional requirements, the properties that the functions must have, such as performance and usability. These requirements are as important as the functional requirements for the product's success. 
5. Project issues, the conditions under which the project will be done. The reason for including them as part of the requirements is to present a coherent picture of all factors that contribute to the success or failure of the project and to illustrate how managers can use requirements as input when managing a project.

VOLERE methodology is an efficient way to describe requirements. In this document focus is on both the user requirements from the perspective of end-user needs and the system requirements that are assumed to be derivative of these former ones as well as other identified ones during the architectural framework. We assume that it is not possible to present only user view on the Hit-Gate project because users are not aware of many important system requirements, constraints, and other network requirements for developing a heterogeneous Hit-Gate Network of Networks (HG NoNs). Here, we will deliver an assemble of overall requirements collection consisting of end-user requirements and some high level functional and nonfunctional requirements in groups (chapters) more related to HitGW and its architecture. They can be divided into the following groups:

- Communication Requirements

- Data Requirements

- Usability Requirements

- Performance Requirements

- Operational Requirements

- Security Requirements

- Legal Requirements.

VOLERE specifies description of particular requirement with following characteristic:

A. Requirement Numbering — give each requirement a unique identifier to make it traceable throughout the development process. It is suggested that the numbering should include:

- Requirement number - the next unique requirement number;

- Requirement Type - the section number from the template for this type of requirement. This field serves as a reminder of what this requirement relates to and helps to compare requirements of the same type leading to detection of contradictions and duplications.

B. Event/use case number - the identifier of a business event or use case that contains this requirement. There might be several event/use case numbers for one requirement because the same requirement might relate to a number of events/use cases.

C. Customer Value - a measure of how much end-user cares about each requirement. It indicates the level of customer (end-user) satisfaction, if a given requirement will be implemented. It may help in prioritizing requirements and then system/solution's functions knowing which of them are the most important and desirable from customers (end-users) point of view. It helps looking at from different perspectives, and to uncover what they care about most deeply.

D. Priority - importance of the requirement.
E. Dependencies - keep track of other requirements that have an impact on this requirement.

F. Conflicts - keep track of other requirements that disagree with this one.

For this research we use simpler requirement cards with elements most suitable in this project. The fields in a questionnaire of the requirement template are:

- 'Id' is an identification of the requirement. It also includes a requirement type that precedes the requirement number. The requirement type is created as an abbreviation of <level $2>$ and $<$ level $3>$ of sections (linked with a dash) that group requirements into types.

- 'Importance (priority)' (High/Medium/Low) tells how a requirement is important for end-users. "High priority" requirements are direct requirements of Hit-Gate, "Medium priority" ones are only desired requirements, and "Low priority" ones are optional.

- 'Source' indicates to references to a given requirement.

- 'Version' shows the evolution of the requirement.

- 'Speed' (Normal/Urgent) tells about a need of availability of the requirement in time during a mission.

- 'Reliability' (Normal/High) specifies the reliability of implementation of a given requirement. "High" means that there should be a confirmation of receipt of communication.

- 'Auditable' (Normal/High) denotes that the requirements should be tracked during operation.

- 'Security' (Standard/Enhanced) gives a level of security that the requirement should have.

- 'Quality' (Normal/High) tells about the quality of performing the requirement.

- 'Title' is a requirement name.

- In the 'Description' field the requirement is explained.

The process of defining end-users' requirements for the Hit-Gate project is split into four stages. Using existing different sources delivered by partners and found in available bibliography resources the preliminary list of requirements is collected in the stage I (from e.g. [1], [4], [5], [6], [7], [9]). These requirements are then updated by partners basing on their knowledge and experience in the stage II. In the stage III end-users are involved to review existing requirements and to define new ones. It is done during meetings at national level organized by consortium's partners. Then the collected information is merged in the stage IV.

\section{GENERAL OBJECTIVES}

The Hit-Gate project defines the following general objectives for the HitGW that can be treated as baseline requirements [4]:

O1 it shall enable communications between FirstResponders' heterogeneous networks used at European Level;

$\mathrm{O} 2$ it shall provide set of services required to meet FirstResponders' needs across First-Responders' heterogeneous networks; 
O3 it shall provide cross-network services to FirstResponders in a seamless way;

O4 it shall be secure and shall comply with pre-established security policies;

O5 it shall be transportable, rapidly deployable, and autonomous;

O6 it shall comply with mission critical requirements;

O7 it shall automatically integrate new networks of known type;

O8 it shall require minimal or no changes to existing public safety communication (PSC) infrastructures;

O9 it shall provide a modular architecture allowing incorporation of future network types;

O10it shall provide open-interfaces and will provide recommendations for standards;

O11it shall address recommendations for interoperability dealing with operational, organizational, and legal aspects.

O12it should ensure information exchange among FirstResponders' networks.

The underlying requirements are [2]:

- Communications connectivity,

- Communications reliability,

- Communications deployability,

- Communications transparency: i.e. end-users are blind to the presence of communications, and only interact amongst each other at the information layer on the basis of "send information and forget how it gets there".

\section{V.END-USER REQUIREMENTS AND NEEDS}

Identified functional requirements are shown in Table I.

Identified nonfunctional requirements (e.g. quality, efficiency, etc.) related to provided services are:

- for voice: Clarity of communications (no noise); High quality voice codecs;

- for data: Broadband data access; Enabling carry peak data rate.

The most important requirements identified in the process described in Section III are:

- PSC system in a typical operation should ensure connectivity Scene Command (tactical level) with Incident Command and with data centers (operational level) following the chain of command;

- There is a need to establish standard operating procedures;

- The interoperability gateway should not limit the coverage of communication provided by interoperating radio systems used on site during mission to an incident;

- The gateway should support from legacy to current to next-generation broadband networks used today by FRs;

- End-users of all wireless systems should have mobility as offered by a given system used on the scene;

- Interoperability gateway shall be developed in a way that allows adding new network (of known type) in a modular way, therefore ensuring an always up-to-date gateway;
- Interoperability gateway shall provide open-interfaces and a wide range of services across networks, based on widely adopted standards. These include IP (Internet Protocol) as basis protocol (for level 3 OSI interoperability), IPTV, VoIP, (Voice over IP), and SIP/SDP (Session Description Protocol) for signaling;

- $\quad$ PSC system in a typical operation should ensure inter communication between different entities, and these entities with Scene Command;

- Interoperability gateway should enable communications to FRs during operations across heterogeneous networks, using their current receiver equipment and with no impact on currently used PSC base-stations and infrastructures other than providing a connection-point to interoperability gateway;

- Interoperability gateway should automatically integrate new networks of known type using such functionalities like: (i) 'plug'n'play', being the ability for new networks (of known type) to be connected to interoperability gateway and become ready to use; and (ii) 'on-the-fly', being the interoperability gateway ability to automatically adapt and work with newly connected networks (of known type) without need to temporarily stop services;

- Interoperability gateway should be developed adopting methodologies defined for critical security systems, shall use secure technology and shall use proven security techniques for comprehensive end-to-end security across all networks;

- $\quad$ PSC system in a typical operation should ensure intra communication in particular entities — in Local Headquarters and between Local Headquarters and Remote Headquarters;

- There is a need for a set of standards for public safety services.

The most important requirements with their score are shown in Fig. 1.

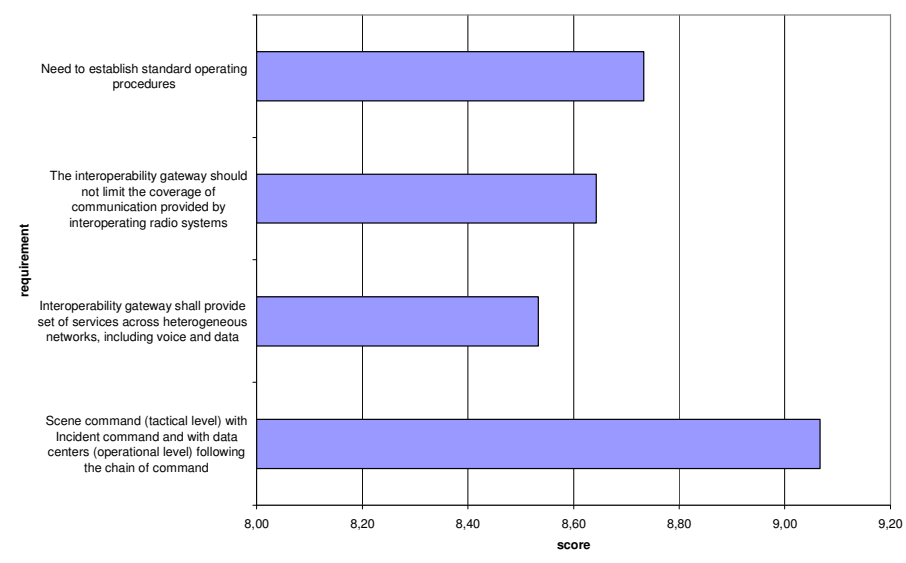

Fig. 1. Score for identified preliminary needs 
Table I. Functional requirements

\begin{tabular}{|c|c|}
\hline $\begin{array}{c}\text { Do- } \\
\text { main }\end{array}$ & Functional requirements \\
\hline 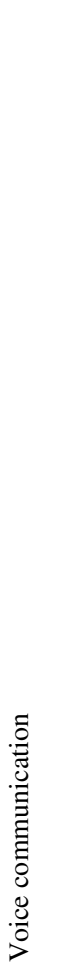 & $\begin{array}{l}\text { Internal voice communications such as between closed user } \\
\text { groups and end-to-end communications, etc.; } \\
\text { External voice communications with other public safety } \\
\text { forces or emergency services, at local level; } \\
\text { Group communication: } \\
\text { - ensures one to many communication to all registered in } \\
\text { the group, regardless of the number of networks involved } \\
\text { in the transmission, as well as the number of active users } \\
\text { - must prevent the unauthorized access to a group } \\
\text { - } \quad \text { provides automatic answer of all calls within the group } \\
\text { - } \quad \text { prevents simultaneous voice transmission of many users } \\
\text { within the same group } \\
\text { - } \text { should notify the user about crossing the border of } \\
\text { configured in the system group's activity area } \\
\text { ensures broadcasting capabilities to all registered users of } \\
\text { the group } \\
\text { should provide a user ID presentation for all users of the } \\
\text { group, regardless of the network used; } \\
\text { Emergency calls to the number } 112 \text {; } \\
\text { Calls for end-user's PBX network; } \\
\text { Call recording in dispatching communication system; } \\
\text { Capability of assigning priorities to the individual users and } \\
\text { type of connection; } \\
\text { Capability of connection queuing }\end{array}$ \\
\hline $\begin{array}{l}\tilde{\Xi} \\
\tilde{\Xi} \\
0\end{array}$ & $\begin{array}{l}\text { Data access to radio channel; } \\
\text { Data access for querying or others databases; } \\
\text { Data transmission for decision making; } \\
\text { Data exchange between different First-Responders; } \\
\text { Image transmission if possible; } \\
\text { Video transmission if possible; } \\
\text { Metadata transmission for smart surveillance }\end{array}$ \\
\hline$\stackrel{\bar{\Xi}}{\stackrel{5}{0}}$ & $\begin{array}{l}\text { Roaming capabilities for cross-border operation if systems } \\
\text { support this feature; } \\
\text { Wide range of possible compatible communication } \\
\text { technologies operating; } \\
\text { Interoperable ad-hoc mesh network for several First- } \\
\text { Responders operating in the same coverage area including } \\
\text { other mesh networks deployed; } \\
\text { Remote management of terminal by systems administrator } \\
\text { (radio and telephone); } \\
\text { Retransmission }\end{array}$ \\
\hline
\end{tabular}

\section{The Hit-Gate ARChitectural CONCEPT}

The Hit-Gate architecture (Fig. 2) is based on the basic concept of a Network of Networks ([15], [16]) to create a comprehensive system that supports Cross-Network Services (CNSrv) among FRNs. The Hit-Gate system is based on IP-based transmission, routing, signaling and communication. The system is compatible with any IMS type of network [17] that had been adapted in some key standards, e.g., 3GPP $\left(3^{\text {rd }}\right.$ Generation Partnership Project), 3GPP2 and ETSI (European Telecommunications Standards
Institute) TISPAN (Telecommunications and Internet converged Services and Protocols for Advanced Networking). IMS was selected to be implemented in the core of the Hit-Gate system because it performs all functions needed for call switching, user authorization and development of new services. Instead of development of an own switching and control node that manages calls and value-added services, it would be more efficient to use an existing and proved solution like IMS because it provides flexibility and ease for software developers.

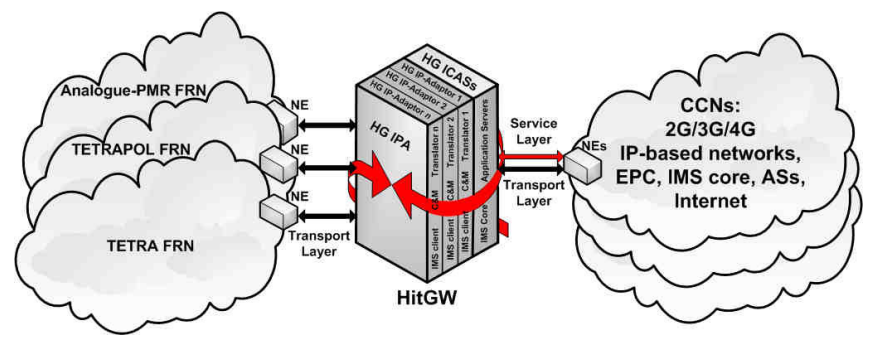

Fig. 2. The Hit-Gate architectural concept

One of the main components of the system gateway is HG IMS Core and Application Servers (HG ICASs) subsystem that is responsible for control, signaling and the CNSrv. The HG IMS Core functions are related to the generic communications services and standardized IMS interfaces, including IMS-based services. This component provides the basis for interoperability since it defines a common framework that ensures the interworking of all system nodes. The HG Application Servers (ASs) in the HG ICASs allow seamless access to CNSrv (e.g. presence function and conference connections) across whole HG NoNs even under high-dynamic conditions when additional NEs of the FRNs are attached using 'on-the-fly' and 'plug'n'play' features. The HG ICASs supports also transport network management (configuration, translation of data and protocol as well as discovery of network nodes) and transmission security.

The FRNs are connected to HG ICASs with HG IPA that consists of a set of $n$ HG IP-adaptors $(i=1, \ldots, n)$. To have an access to a FRN over its access point, a FR Mobile Terminal (FR-MT) or Base Station (BS) and an HG IP-adaptor is required. A FR-MT is connected using manufacturer's specific interface by wire with HG IP-adaptor. FR BS can be also used instead of a FR-MT. The HG IPA can be done as one compact device that even allows connecting several incompatible technology types of mobile terminals or base stations, or it can be deployed as a distributed sub-system of $n$ HG IP-adaptors and their attached FR-MTs or BSs. In this case the HG IPA and HG ICASs can be implemented on rugged PC tablets or laptops, respectively. An HG IP-adaptor consists of: an IMS Client representing unique user identification number; FR technology Translator that is responsible for translating the information from FR-MT into appropriate IMS messages as 
well as from IMS messages into commands understood by FR-MTs; and Control and Management (C\&M) component needed by an dispatcher who can handle CNSrvs among FRNs.

The "clouds" of incompatible FRNs and CCNs are interconnected with the HitGW. Thus a HG NoNs is created for CNSrv between FRs, its agencies, and other entities involved in a disaster scenario that used IP-based networks. The application components can be distributed and provided from HG ICASs or any CCN compatible with IMS, e.g. Evolved Packet Core (EPC).

The HG IPA constitutes a form of "bridge" between HG ICASs and specific radio access technologies that use manufacturer proprietary protocols which are accessible with an Application Programmable Interface (API) of the FR-MTs (Fig. 2). A HG IP-adaptor of HG IPA is used to adapt a NE of one FRN to an IMS type of network where services are controlled by HG ICASs. Due to this adaptation the IP connectivity is achieved between the FR-MTs and other IP NEs of the HG NoNs. Two types of interfaces are used to connect a HG IP-adaptor and HG ICASs:

i) SIP with extended header between HG IMS Client and HG ICASs (IMS interface); and

ii) HTTPS (Hyper Text Transfer Protocol Secured) for control and management commands (C\&M interface).

The HG IP-adaptor has two additional interfaces:

i) External: to the mobile terminal; this interface has to meet radio manufacturer's specification (Translator specification).

ii) Internal: between HG IMS Client, C\&M and Translator (Commands interface).

The HG IP-adaptor was designed to be compatible with several communication technologies listed in section II. It was developed comprising the common interfaces and functions used by the IMS type of network. For development of HG IP-adaptors for a FRN a software template in C++ for Visual Studio is available that can be extended to have a new HG IP-adaptor.

\section{Hit-GATE High LEVEL ARCHITECTURE}

\section{A. Development framework}

In order to meet the basic requirements of creating an interoperable HG NoNs (see section IV), and all end-users requirements that are very diverse in application domains and configurable quantities, the HitGW should be based on a solution of significant flexibility. To achieve it several modern techniques have been selected to create a holistic development framework. The innovativeness of the gateway under the design makes use of the IP domain operating with SIP, IMS, and other open source solutions like On-Demand Intelligent Network Interface (ODINI) [14] that allow integrating e.g. trunking/dispatching analog/digital systems as well as data transmission ones. Such an approach accelerates a process of establishing the communication among different emergency services and other governmental organizations during crisis because the configuration procedures can be well-defined and versatile.

For the development framework of the Hit-Gate High Level Architecture (HG HLA) the Open IMS Core (www.openimscore.org) by Fraunhofer FOKUS and the application server Mobicents (www.mobicents.org) were selected. It is open source software that offers a flexibility to develop the IMS services and extensible solution and enables to make a proof of concept. The VoIP communicators were softphones OpenIC_lite for Ubuntu and X-Lite for Windows / Mac OS X. The Mobicents application server used JBoss server and SIP Servlets technology.

The HG HLA developed in Hit-Gate is shown in Fig. 3. This architecture has two layers: transport and service. The control/signaling sub-layer of the service layer consists of three Call Session Control Function (CSCF) modules and Home Subscriber Server (HSS) where subscribers' profiles are stored. The HG ASs lie on the application/service sublayer of the service layer where the services are executed to provide them to the end-users.

The HG IP-adaptors (a distributed system solution of the HG IPA) connect access NEs of FRNs or CCNs to the HitGW, and jointly with HG ICASs they create a HG NoNs of incompatible NEs from FRNs and CCNs that are capable to exchange and use the information carried among them and thus offering the CNSrv for FRs. It ensures flexible and quick attachment of PSC systems.

Proxy CSCF (P-CSCF) component is a server that is the first point where a call is received. It also serves a function of firewall at the application level. Serving CSCF (S-CSCF) component is a central node responsible for handling the signaling. Interrogating CSCF (I-CSCF) deals with incoming SIP calls. Its address is published via DNS of the domain. It allows other servers to find this node and use it as a router for SIP packets. HSS is a main database about subscribers that supports call handling.

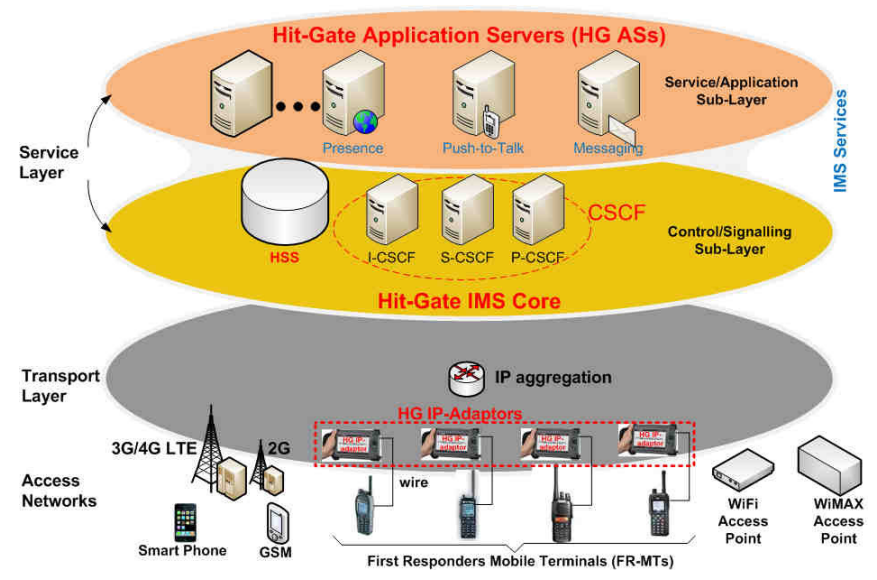

Fig. 3. The Hit-Gate High Level Architecture view 
The architecture presented in Fig. 3 allows creating a wide scope of different services. FRs' services were primary targeted. The following services have been implemented: registration and authentication of NEs, presence, full-duplex and half-duplex private and group calls, emergency call, messaging, calling/talking party identification, broadcast call, retrieving a group list, group advertisement and session modification. Usage of other services (e.g., images, video streaming, IPTV) that are typical for $2 \mathrm{G}, 3 \mathrm{G}$ and $4 \mathrm{G}$ LTE type of networks can be performed as well between access NEs of FRNs and NEs of CCNs.

\section{B. GSM Testbed}

In a testbed developed we implemented the IMS platform to work with GSM (Global System for Mobile communication) network and to deliver voice communication and SMS (Short Message Service) between mobile terminals and a dispatcher. We used Nokia N900 mobile phone as a gateway terminal (access NE) to a GSM network. The analog voice signal from/to the terminal was processed in AD/DA converter and forwarded digitally with USB port to RTP driver and then to SIP softphone client. The mobile terminal was controlled using another USB port and by a software being an extension of the SIP client.

The testbed architecture is shown in Fig. 4. In such a testing environment we could set up voice calls between end-users' 2G/3G terminals and dispatcher's softphone client. Both call parties could also send SMS texts each other.

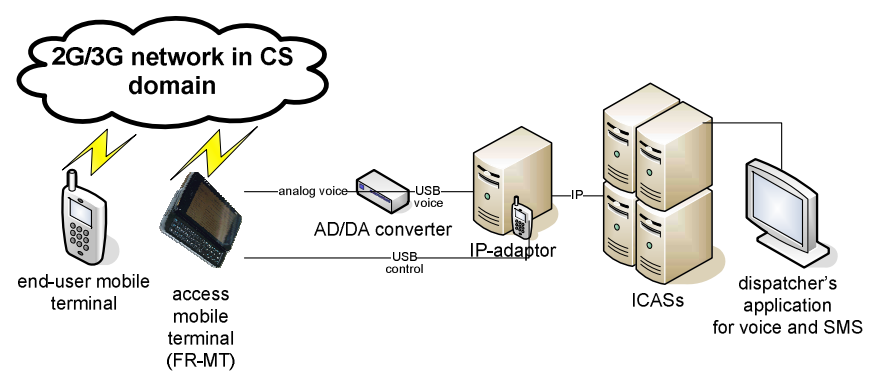

Fig. 4. Block diagram of the IMS testbed

\section{VIII.CONCLUSIONS}

In Fig. 5 one can see the coverage of end-user requirements with requirement types. The majority of requirements concerns communication aspects, and then performance ones.

Hit-Gate user requirements can be divided into five main categories of requirements:

C1 Communication services and performances requirements,

C2 Mobility and connectivity requirements,

C3 Interoperability requirements,

C4 Security and safety (i.e. confidentiality, availability, integrity) requirements,
C5 Management as well as monitoring needs requirements, from which category $\mathrm{C} 1$ and $\mathrm{C} 2$ are described with the greatest number of requirements during the process of requirement collection.

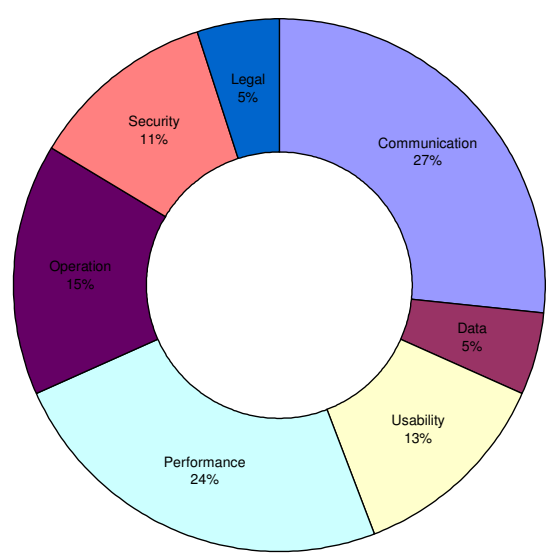

Fig. 5. Coverage of end-user requirements with requirement types

The HitGW that offers interoperability between incompatible NEs of the HG NoNs has to meet end-user needs and expectations. Identified and defined requirements will be used for the future Hit-Gate product developments and as the guidelines for the Next Generation Networks (NGNs) and Future Mobile Convergence (FMC) as well as for ongoing standardization activities. They will be also helpful in defining overall and detailed system architecture, preparing system specification, as well as describing scenarios and use-cases, e.g., for the demonstration purposes.

The HG HLA is based on the NoNs concept. The system gateway HitGW is composed of two sub-systems: HG IPA and HG ICASs. Both sub-systems can be used remotely connected as network elements of HG NoNs or integrated as one compact system solution that interconnects incompatible NEs of the FRNs and IP-based networks. Within a HG IP-adaptor each FR technology (that is accessible via a FR-MT) should have a suitable technology translator that constitutes an interface between an access NE of the FRN and HitGW. The other HG IP-adaptor components - IMS Client and C\&M - provide service as well as configuration and management solutions for FR-MT attached to it as well as for itself. Such an approach facilitates interoperability between heterogeneous systems because one just needs to develop a new translator in order to allow connectivity of a new FR-MT to its FRN.

The HG HLA was applied to elaborate a demonstrator that allowed developing a HitGW prototype as an interoperability gateway that offers FRs services and other services provided by $2 \mathrm{G} / 3 \mathrm{G} / 4 \mathrm{G}$ networks. The proposed system solution based on IMS occurred easy to install, run and configure. Development of new services to integrate other 
communication systems needs Java programming and knowledge about API commands and hardware interface to control the communication via the terminal attached to the gateway. New services based on the IMS platform can extend the communication capabilities among PPDR entities offering them even ad-hoc integration and management.

\section{REFERENCES}

[1] "D2.1, Case studies and scenarios report," Hit-Gate project, 2012.

[2] "Deliverable D2.2, Analysis of crisis management system requirements, ver. 3," SECRICOM project, July 2009.

[3] "Requirements specification template," Edition 15 March 2010, James \& Suzanne Robertson principals of the Atlantic Systems Guild.

[4] "Description of work of the Hit-Gate project," 2011.

[5] "Report of the workshop on 'Interoperable communications for safety and security' ", Workshop jointly organized by DG ENTR and DG JRC with the support of EUROPOL and FRONTEX, Gianmarco Baldini, 28-29 June 2010 - Ispra, Italy, EUR 24540 EN

[6] "PPDR spectrum harmonisation in Germany, Europe and globally," WIK-Consult, Final Full Public Report, Bad Honnef, 6 December 2010.

[7] "LTE as potential communications technology for public safety operations - a usability study taking into account the aspect 'private vs. shared network' ", Baccalaurean Thesis II, Ing. Manfred BLAHA, 2011.

[8] "Meeting the challenge: the European security research agenda," European Security Research Advisory Board, September 2006 http://ec.europa.eu/enterprise/policies/security/files/esrab-re-porten.pdf.

[9] S. O'Neill et al., "User requirements for mission-critical application the SECRICOM case," Technical Sciences, No. 15(1)/2012, 2012.

[10] W. Wojciechowicz et al., "Seamless communication for crisis management," Technical Sciences, No. 15(1)/2012, 2012.
[11] W. Wojciechowicz et al., "Information and communication technology and crisis management", Technical Sciences, No. $15(1) / 2012,2012$.

[12] ETSI TS 102 181, "Emergency communications (EMTEL): Requirements for communication between authorities/organizations during emergencies," 2008.

[13] Network Centric Operations Industry Consortium, "Findings and recommendations for mobile emergency communications interoperability (MECI)," 2007.

[14] Rohill, "Whitepaper, On-Demand Intelligent Network Interface," 2009

[15] "D3.4, Hit-Gate High-Level Architecture and Interface Control - Final Version," Hit-Gate project, 2013.

[16] "D4.5, Generic Interoperability Framework: Interfaces and Functions - Final version," Hit-Gate project, 2014.

[17] "D4.10, Hit-Gate System (HW and SW): Design, Source Code, Binaries and Unit Test Results Iteration 2 - Final," Hit-Gate project, 2014.

[18] www.hit-gate.eu

[19] Thomas Weber, "Public Safety Future Networks - European Update," Future Network \& Mobile Summit 2013, 4 July 2013

[20] ECC Recommendation (08)04, "The identification of frequency bands for the implementation of Broad Band Disaster Relief (BBDR) radio applications in the $5 \mathrm{GHz}$ frequency range," October 2008

[21] ECC Recommendation (11)09, "UWB Location Tracking Systems," October 2011

[22] WGFM, "Implementation Roadmap for the Mobile Broadband applications for the Public Protection and Disaster Relief (PPDR)," Draft

[23] ECC Report 199, "User requirements and spectrum needs for the future European broadband PPDR system (Wide Area Network)," Draft

[24] ECC FM49(12)017, "FM 49 Radio Spectrum for PPDR," 20-21 March 2012 